\title{
Displacement of Retained Subretinal Perfluorocarbon Liquid Through Therapeutic Retinal Detachment Induced by Balanced Salt Solution Injection
}

This article was published in the following Dove Press journal:

International Medical Case Reports Journal

\author{
Marc Saab \\ Simon Javidi $\mathbb{1 D}^{2}$ \\ Ali Dirani $\mathbb{1}^{3}$ \\ Ghassan Cordahi ${ }^{2}$ \\ 'Department of Ophthalmology, Hôpital \\ Charles LeMoyne, Université de \\ Sherbrooke, Longueuil, Québec, Canada; \\ ${ }^{2}$ Department of Ophthalmology, Hôpital \\ Maisonneuve-Rosemont, Université de \\ Montréal, Montréal, Québec, Canada; \\ ${ }^{3}$ Department of Ophthalmology, CHU de \\ Québec - Université Laval, Québec. \\ Québec, Canada
}

Correspondence: Simon Javidi

Department of Ophthalmology,

Université de Montréal, Montréal,

Québec, Canada

Email simon.javidi@gmail.com
Purpose: To report a case of displacement of retained subretinal perfluorocarbon liquid (PFCL) through therapeutic retinal detachment (RD) induced by balanced salt solution (BSS) injection. Methods: This is a surgical case report. We present a case of a 61-year-old woman who presented with subretinal PFCL at the papillo-macular bundle with best-corrected visual acuity (BCVA) of 20/200 at four weeks following RD surgery in her right eye. She underwent a three-port pars plana vitrectomy with therapeutic RD of a portion of the posterior pole and inferior periphery induced by BSS injection, followed by complete air-fluid exchange, and kept an upright position for three days to force the displacement of the PFCL bubble towards the inferior retinal periphery. BCVA assessment, dilated fundus examination (DFE), and optical coherence tomography (OCT) were performed before and after the surgery.

Results: Two weeks after the procedure, BCVA improved to 20/40, the subretinal PFCL was not visible on DFE, and a control OCT confirmed displacement of the PFCL bubble with atrophy at the papillo-macular bundle. There were no complications.

Conclusion: Displacement of retained subretinal PFCL through therapeutic RD induced by BSS injection seems to be an effective technique with fewer potential complications in comparison to the more traditional approach of removal by direct aspiration.

Keywords: perfluorocarbon liquid, subretinal perfluorocarbon liquid, vitreoretinal surgery, complication, retinal detachment

\section{Plain Language Summary}

Perfluorocarbon liquid (PFCL) is often used in retinal detachment surgery. Sometimes, the PFCL can pass through a retinal tear and get stuck under the retina. If this happens close to the part of the retina responsible for central vision (macula), it can cause significant loss of vision due to mechanical effects and toxic effects. In those cases, the subretinal PFCL cannot be left under the retina. Traditionally, the PFCL is removed directly (aspirated) if needed. In this article, the authors describe a surgical case where they did not remove the PFCL, but instead, successfully displaced it towards a less critical area of the retina in order to protect the macula. Although this technique is not new, it is little known and, theoretically, might be less risky than the traditional approach of direct removal.

\section{Introduction}

Retained subretinal perfluorocarbon liquid (PFCL) is a known complication of using PFCL in retinal detachment (RD) surgery. This complication may considerably reduce 
visual acuity (VA) if it occurs near the macula. Moreover, if the PFCL reaches the subfoveal area, it can cause local damage to the retinal pigmented epithelium (RPE) and photoreceptors, leading to permanent decrease in VA. ${ }^{1}$ Current evidence suggests removal of subretinal PFCL if VA is significantly impaired. ${ }^{2}$ As concluded by Liu et $\mathrm{al}^{2}$ in their review of the management of subfoveal PFCL, no specific technique is currently recommended, mainly because of the lack of randomized or controlled trials, the limited number of cases reported, combined with the uniqueness of each case.

\section{Case Report}

A 61-year-old woman consulted initially for reduced vision in her right eye with a best-corrected visual acuity (BCVA) of "counting fingers". "Fundus examination showed a temporal bullous macula-off retinal detachment with several peripheral large retinal tears."

Accordingly, the patient underwent a three-port pars plana vitrectomy (PPV) with PFCL injection (CBausch \& Lomb, DK-Line ${ }^{\circledR}$ PFCL, Rochester, New York, United States), followed by laser treatment around the peripheral retinal tear edges, PFCL-air exchange, and silicone oil (5000 cSt) injection for endotamponade. Of notice that silicone oil was used because the patient had a cervical herniated disc and post-surgical positioning was not possible at the time. The patient subsequently developed a rapid progression of her cataract. Four months after the first surgery, the patient underwent combined phacoemulsification-PPV surgery with removal of silicone oil. The retina was inspected and was flat over 360 degrees.

One month after the last surgery, the patient reported reduced vision with BCVA of 20/200. The dilated fundus examination (DFE) and optical coherence tomography (OCT) showed a subretinal PFCL bubble located under the papillo-macular bundle (see Figure 1A). Consequently, two weeks later, a three-port PPV was performed to displace the bubble with the following procedure (see Video, Supplemental Digital Content 1, which demonstrates the salient aspect of this technique). Retinotomies were made near the inferotemporal arcade on the nasal side and inferiorly (see Figure 2A) with a 41-gauge De Juan cannula and balanced salt solution (BSS) was injected subretinally. The vitrectomy machine was set for an automatic infusion/ injection rate of $10 \mathrm{mmHg}$. The injected subretinal BSS created a serous RD that formed a pocket right under the inferotemporal arcade, which spread to reach the PFCL bubble (see Figure 2B). The purpose of this pocket is to enable the displacement of the PFCL bubble away from the papillo-macular bundle. The retina was thoroughly reexamined to ensure the absence of peripheral tears. Then, a complete air-fluid exchange was performed. Of note, the displacement of the PFCL bubble did not take place during the surgery; the patient had to remain in the upright seated position for three days to force the displacement of the

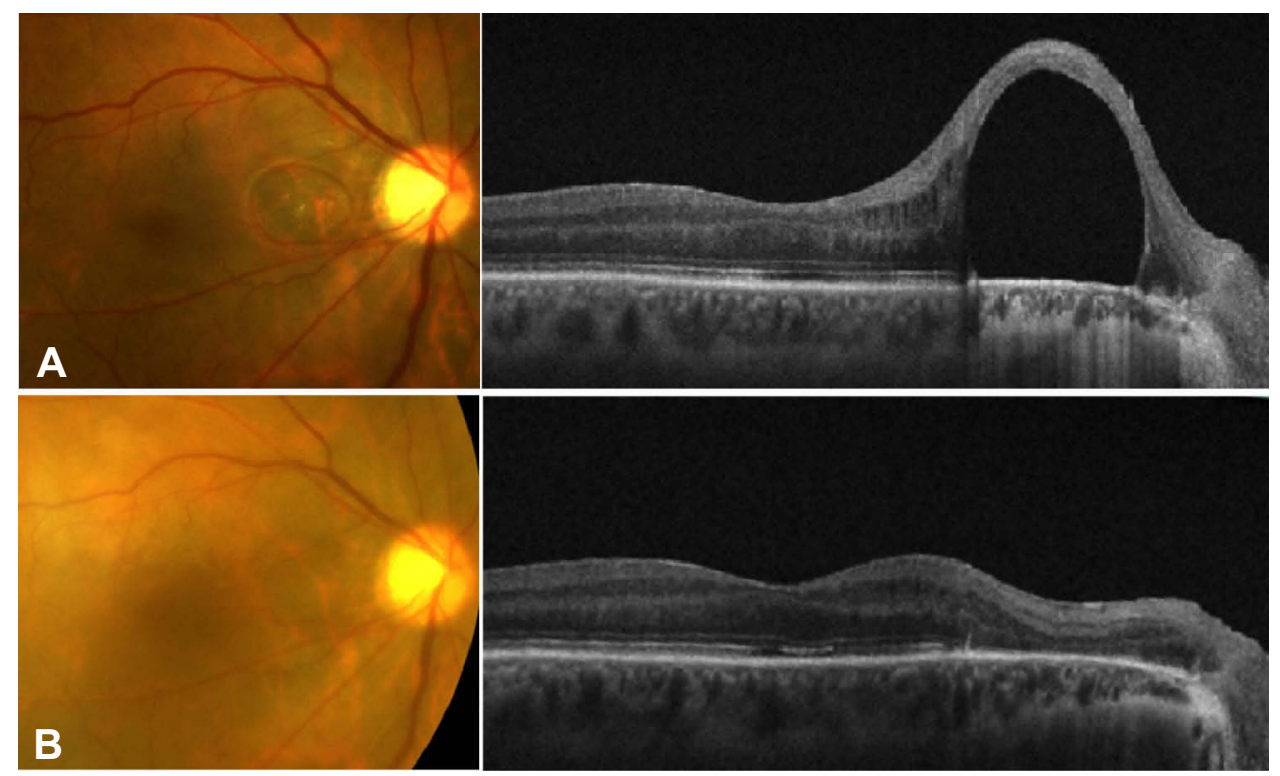

Figure I (A) Preoperative fundus photograph (left) and optical coherence tomography (OCT) image (right) showing a subretinal perfluorocarbon liquid (PFCL) bubble located under the papillo-macular bundle, due to a complication of retinal detachment (RD) surgery. (B) Fundus photograph (left) and OCT image (right) 2 weeks posttherapeutic inferotemporal RD induced by balanced salt solution injection, confirming displacement of the subretinal PFCL bubble with atrophy at the papillo-macular bundle. 

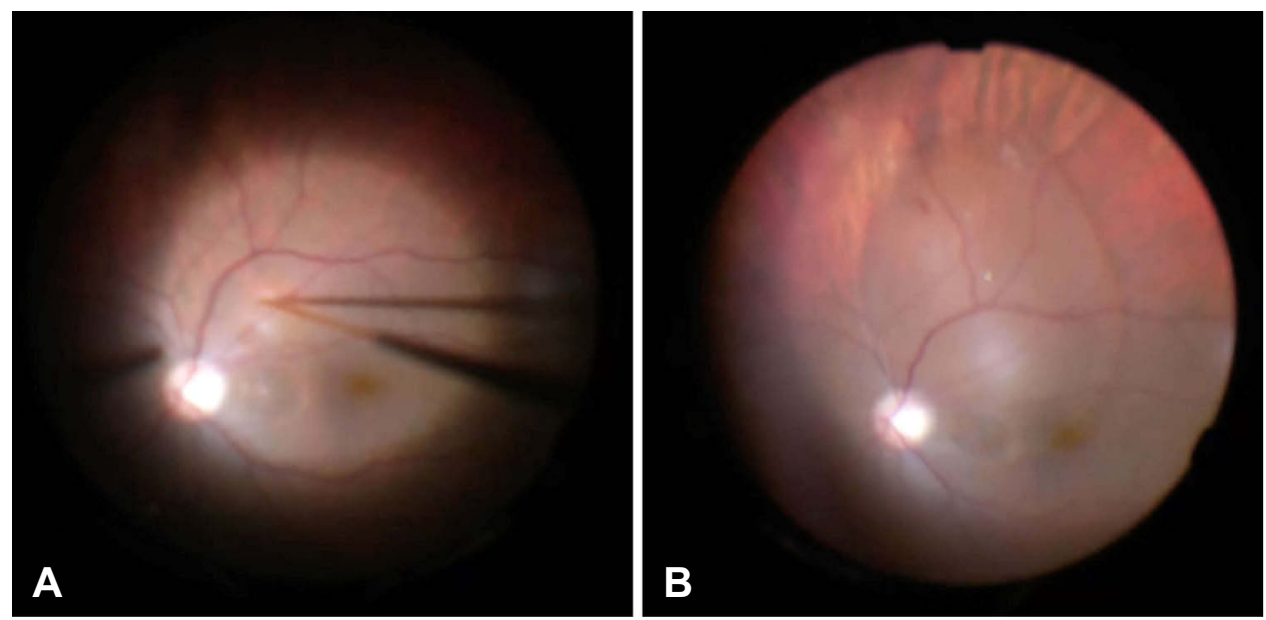

Figure 2 Intraoperative photographs. Before (A) and after (B) subretinal balanced salt solution (BSS) injection using a 4 I-gauge De Juan cannula. Subretinal BSS created a serous retinal detachment that formed a pocket right under the inferotemporal arcade, which spread to reach the perfluorocarbon liquid bubble.

bubble towards the inferior retinal periphery. The surgery had no complications. Two weeks after the procedure, BCVA improved to 20/40, the subretinal PFCL was not visible on DFE, and a control OCT confirmed displacement of the PFCL bubble with atrophy at the papillomacular bundle (see Figure 1B). Of note, we expected to locate the subretinal PFCL bubble in the inferior retinal periphery and treat it with argon laser barricade. Although this was our original intent, we could not locate any subretinal PFCL post-operatively using indirect ophthalmoscope with scleral depression.

\section{Discussion}

Retained subretinal PFCL is a complication that can generally occur in up to approximately $11 \%$ of vitreoretinal surgeries requiring intraoperative PFCL. ${ }^{3}$ The two risk factors that seem to be considerably associated with this complication are peripheral retinotomies larger than 120 degrees and insufficient saline rinse after removal of PFCL. ${ }^{3}$ Garcia-Valuenza et $\mathrm{al}^{3}$ found subretinal PFCL in $40 \%$ of eyes with a 360 -degree retinotomy.

Subretinal PFCL can cause acute vision loss if located near the macula. Furthermore, in the long run, submacular PFCL is toxic and causes retinal damage due to an inflammatory reaction involving macrophages with intracellular vacuoles containing PFCL. ${ }^{1}$ However, subretinal PFCL seems to have no impact on VA if located far from the macula. ${ }^{2}$ Therefore, the technically challenging removal of retained PFCL is recommended depending on its location and impact on vision, with the intent of restoring VA and preventing irreversible damage to the macular anatomy. ${ }^{2}$
In our case, the patient probably had subretinal PFCL under the papillo-macular bundle for several weeks before it was displaced towards the inferior retinal periphery; BCVA went from 20/200 to 20/40 just two weeks after the procedure, suggesting no severe irreversible damage occurred in that short timeframe.

Several techniques are mentioned in previous case reports, including direct aspiration of the PFCL bubble, displacement of the PFCL bubble with its subsequent removal, and displacement of the PFCL bubble without its removal. ${ }^{2}$ The most reported method is surgical aspiration through a retinotomy that is either next to the PFCL bubble, or directly on top of it, using a small-gauge cannula (ranging from 25 - to 50 -gauge). ${ }^{2}$ Reported complications due to the risk of trauma with the direct aspiration technique include macular hole, irreversible alteration of the RPE, and submacular hemorrhage. ${ }^{4}$

In a retrospective case series of three patients, Le Tien et $\mathrm{al}^{5}$ reported a novel surgical technique to displace subfoveal PFCL without removing it from the subretinal space. They displaced the subfoveal PFCL droplets towards the inferior retinal periphery by creating a therapeutic RD using BSS injection during 20-gauge PPV, a technique very similar to ours. ${ }^{5}$ They made their therapeutic $\mathrm{RD}$ at the posterior pole and inferior periphery by injecting BSS with a 39-gauge flexible cannula through a retinotomy adjacent to the inferotemporal vessels. ${ }^{5}$ All three cases had significant improvement in postoperative BCVA, with no complications. ${ }^{5}$ In contrast, we used a 41gauge cannula to inject BSS during 25-gauge PPV, which reflects the recent advances in technology and the latest 
equipment available at the time in order to minimize undesirable surgical outcomes.

In conclusion, the consequence of retained subretinal PFCL varies depending on the size, anatomic location in regard to the macula, and amount of time it has been in contact with retinal structures. Although the literature is scarce on this topic, in our experience and in accordance to Le Tien et $\mathrm{al}^{5}$, $\mathrm{s}$ work, displacement of retained subretinal PFCL through therapeutic RD induced by BSS injection seems to be an effective technique with less potential complications in comparison to the more traditional approach of removal by direct aspiration.

\section{Summary}

Management of retained subretinal perfluorocarbon liquid (PFCL), a complication of retinal detachment surgery, is recommended if visual acuity is at risk. The authors describe a case where they displaced a subretinal PFCL bubble located under the papillo-macular bundle, instead of removing it via the more traditional approach of direct aspiration.

\section{Authorship}

Each author attests that they meet the current ICMJE criteria for Authorship. All authors made substantial contributions to conception and design, acquisition of data, or analysis and interpretation of data; took part in drafting the article or revising it critically for important intellectual content; gave final approval of the version to be published; agree to be accountable for all aspects of the work.

\section{Consent}

This surgical case report does not contain any identifying details or personal information concerning the patient; however, in compliance with The Committee on
Publication Ethics (COPE) guidelines, consent from the patient was obtained for publication of the content in this report concerning the patient (information, images, and video) in publications of International Medical Case Reports Journal. Institutional approval is not necessary to publish the case details according to the author's institution guidelines.

\section{Acknowledgment}

This paper was presented once, by Marc Saab, MD at the Retina Case Conference Canada 2017. Canadian Ophthalmological Society, Le Westin Montréal, Montréal, Québec, Canada; Oct 28, 2017.

\section{Disclosure}

This case study received no funding or grant support. The authors have no financial interest in the products used in this case study. Marc Saab and Ghassan Cordahi are consultants for Alcon, Bausch \& Lomb, Allergan, Bayer, and Novartis. The authors report no other conflicts of interest in this work.

\section{References}

1. Elsing SH, Fekrat S, Green WR, Chang S, Wajer SD, Haller JA. Clinicopathologic findings in eyes with retained perfluoro-n-octane liquid. Ophthalmology. 2001;108:45-48. doi:10.1016/S0161-6420(00) 00481-4

2. Liu W, Gao M, Liang X. Management of subfoveal perfluorocarbon liquid: a review. Ophthalmologica. 2018;240(1):1-7. doi:10.1159/ 000488118

3. Garcia-Valenzuela E, Ito Y, Abrams GW. Risk factors for retention of subretinal perfluorocarbon liquid in vitreoretinal surgery. Retina. 2004;24(5):746-752. doi:10.1097/00006982-200410000-00010

4. Lesnoni G, Rossi T, Gelso A. Subfoveal liquid perfluorocarbon. Retina. 2004;24:172-176. doi:10.1097/00006982-200402000-00031

5. Le Tien V, Pierre-Kahn V, Azan F, et al. Displacement of retained subfoveal perfluorocarbon liquid after vitreoretinal surgery. Arch Ophthalmol. 2008;126:98-101. doi:10.1001/archophthalmol.2007.2

\section{Publish your work in this journal}

The International Medical Case Reports Journal is an international, peer-reviewed open-access journal publishing original case reports from all medical specialties. Previously unpublished medical posters are also accepted relating to any area of clinical or preclinical science. Submissions should not normally exceed 2,000 words or 4 published pages including figures, diagrams and references. The manuscript management system is completely online and includes a very quick and fair peer-review system, which is all easy to use. Visit http://www.dovepress.com/testimonials.php to read real quotes from published authors. 